\title{
Existence, uniqueness and continuity of solutions of integral equations $\left(^{*}\right)$.
}

Richard K. Miller (i) and George R. Sell ( $\left.{ }^{2}\right)$

Summary. - See Introduction.

\section{1. - Introduction.}

In this paper we shall be interested in the solutions $x(t)$ of a nonlinear integral equation of VolmerRA-type:

$$
x(t)=f(t)+\int_{0}^{t} a(t, s) g(x(s) s) d s .
$$

Our objective here is to present a number of theorems concerning the existence, uniqueness and continuity of solution of (1). Existence, and uniquiness theorems have been extensively studied. We note in particular, the following works; $[2,3,6,7,8,10,11,14]$, as well as the bibliography in M.A. Krasnosel'skn's book [6]. The basic techniques for deriving exsistence and uniqueness criteria consist of certain fixed points theorems (for example, the Schauder-Tychonoff Fixed Point Theorem was used by C. Cordunfand [2] and comparison theorems, (for example J.A. NoHEL [8] and T. SATo [11].) Our Theorem 1, is an existence theorem. It is proved with the SoHAdDER-TyonoNOFF Theorem Theorem 2 is an existence and uniqueness theorem and it is proved by the contraction mapping theorem.

(*) The first author was supported in part by the $A$ ir Force office of Seientific Research, Office of Acrospace Research, United States Air Force under AFOSR Grant No. AF-AFOSR 693-67 and by the National Aeronautics and Space Administration under Contract No. NAS8-11264 and Grand No. NGR 40-00:-015. The second author was supported in part by the National Science Foundation under Grant No. GP-3904 and the United States Army under Contract No. DA-31-124-ARO-D-265.

(1) Division of Applied Mathematics Brown University Providence, Rhode Island.

(2) School of Mathematios University of Minnesota Minneapolis, Minnesota and University of Southern Culifornia Los Angeles, California. 
These two theorems ne prove are very general and they include most of those cited above as special cases. Our results are of interest because of the weak assumptions on the kernel $a(t, s)$. For example, in Theorem 2 we only require that the mapping $t \rightarrow a(t, \cdot)$ be continuous in terms of an appropriate weak*-topology.

As is well known, equation (1) does include the initial-value problem for ordinary differential equations

$$
x(0)=x_{0}, x^{\prime}(t)=g(x(t), t) .
$$

So the theory of the solutions of (1) includes that of (2).

One question which seems to have been overlooked by the researchers in integral equations is: How does the solutions $x(t)$ depend on the terms $f(t)$, $a(t, s)$ and $g(x, t)$ ? For ordinary differential equations, this question has been studied in an important paper [4] by E. KAMKE and this theory for ordinary differential equations has been extended recently by $Z$. OPIaL [9]. We feel that the most significant results in this paper are Theorems 3 and 4 which say that the solutions $x(t)$ of (1) depend continuously on the terms $f, g$ and $a$.

The continuity theorems depend on the topology we place on the terms $f, g$ and $a$. We found, not surprisingly, that if one weakened the topology on the kernels $a(t, s)$ it was necessary to strengthen the topology on the terns $g(x, t)$ in order to preserve the continuity results. Although the conclusions of Theorems 3 and 4 are essentially the same, we found that the technique of proof in each case was entirely different.

The continuity results proved here will play a central role in a forthcoming paper of the authors on the topological dynamics of Volterra integral equations.

\section{Preliminaires.}

Let $W$ be an open set in $R^{n}$ and $I$ an open interval in $R$ containing $O$. Let $|x|$ denote the Euclidean norm on $R^{n}$.

HYPoneEsIs A. - The function $f$ is a continuous function on I with values in $W$.

Hypothesis $\mathrm{B}_{p}$. - Let $p$ satisfy $1 \leqq p<\infty$ and let $g(x, t)$ be a measu. rable function defined on $W \times I$ with values in $R^{n}$ such that

(i) for each $t, g(x, t)$ is continuous in $x$, and

(ii) for each compact set $K \subset W$ and each compact set $J \subset I$ there is a measurable, real-valued function $m(t)$ with $|g(x, t)| \leq m(t)$, for $x \in K$ and $t \in I$ and

$$
\int m(t)^{P} d t<\infty
$$


R. K. MLLLER - G. R. SELL: Existence, uniqueness and continuity, etc.

137

A function $g(x, t)$ that satisfies Hypothesis $B_{p}, 1 \leqq p<\infty$, is said to satisfy a Lipschitz condition if for every pair of compact sets $K, J(K \subset$ $\subset W, J \subset I)$ there is a measureable, real-valued function $k(t)$ with

$$
|g(x, t)-g(y, t)| \leqq k(t)|x-y|, \quad(x, y \in K, t \in J),
$$

and $\int_{J} k(t)^{p} d t<\infty$

For each interval $J$ we define the Banach space $\mathbb{I B}_{p}(J), 1 \leqq p<\infty$, by

$$
\mathfrak{J B}_{p}(J)=\mathfrak{I}_{p}\left(J, R^{n}\right), \quad(1 \leqq p<\infty),
$$

where $\mathfrak{I}_{p}\left(J, R^{n}\right)$ is the Lebesgue space of all measurable functions $x$ defined on $J$ with values in $R_{n}$ with $/|x|^{p} d t<\infty$. We shall let $\mathfrak{B B}_{p}^{*}(J)$ denote the adjoint spaces. By a well-known result one has $\mathfrak{J B}_{p}^{*}(J)=\mathfrak{H}_{q}(J)$ if $1<p<\infty$ and $p^{-1}+q^{-1}=1$.

Hyponhesis $C_{p}$. - Let $p$ satisfy $1 \leqq p<\infty$ and let a(t, s) be a mapping of $I \times I$ into the space $M^{n}$ of linear operators on $R^{n}$ such that

(i) for each compact intercal $J \subset l$ and each $t$ in $I$ the mapping $S: \mathfrak{H B}_{p}(J) \rightarrow R^{n}$ defiend by

$$
S: x \rightarrow \int_{J} a(t, s) x(s) d r
$$

is a bounded linear mapping, and

(ii) the mapping $t \rightarrow a(t, \cdot)$ is continuous in the norm topology on $\mathfrak{J B}_{p}^{*}(J)^{n}$.

We shall say that $a(t, s)$ satisfies Hypothesis $C_{p}^{*}, 1 \leqq p<\infty$, if the condition (ii) is replaced by:

$\left(\mathrm{ii}^{*}\right)$ The mapping $t \rightarrow a(t, \cdot)$ is continuous in the weak*-topology on $\mathrm{JB}_{p}^{*}(J)^{n}$.

Hypothesis $C_{p}$ and $C_{p}^{*}$ needs some explanation. If we consider the points in $R^{n}$ as column vectors and the points in $M^{n}$ as square matrices, then (i) can be reformulated as: for every $t$ in $I$, each row of $a(t, \cdot)$ is an element of $\mathfrak{B B}_{p}^{*}(J)$. We then can view $a(t, \cdot)$ itself as an element of the direct sum

$$
\mathfrak{J B}_{p}^{*}(J)^{n}=\mathfrak{H B}_{p}^{*}(J) \oplus \ldots \oplus \mathfrak{J B}_{p}^{*}(J)
$$

for every compact interval $J \subset I$. The weak*-topology, or the norm topology, on $\mathfrak{S B}_{p}^{*}(J)^{n}$ is induced, respectively, by the weak ${ }^{*}$-topology, or the norm topology, on each component. It is clear that Hypothesis $C_{p}$ implies Hypothesis $C_{p}^{*}$ : 
Hypothesis $C_{p}$ or $C_{p}^{*}$, together with Hölders inequality, means that we can find norms on $R^{n}$ and $M^{n}$ so that

$$
\left|\int_{J} a(t, \mathrm{~s}) x(s) d s\right| \leqq\left\{\int_{J}|a(t, s)|^{q} d s\right\}^{\eta / q} \cdot\left\{\int_{J}|x(s)| p d s\right\}^{1 / p},
$$

if $1<p<\infty$ and $p^{-1}+q^{-1}=1$; if $p=1$,

$$
\left|\int_{J} a(t, s) x(s) d s\right| \leqq\|a(t, \cdot)\|_{\infty} \cdot \int_{J}|x(s)| d s
$$

where $\|a(t, \cdot)\|_{\infty}=$ ess. $\sup \{|a(t, s)|: s \in J\}$.

The continuity of the mapping $t \rightarrow a(t,$.$) implies that if t$ is restricted to a compact set $J^{\prime}$ in $I$ then the set

$$
\left\{a(t, \cdot): t \in J^{\prime}\right\}
$$

is a compact set in respectively, the norm topology, or the weak*-topology on $\mathrm{HB}_{p}^{*}(I)^{n}$. This means that

$$
\sup _{t \in J^{\prime}}\left\{\int|\alpha(t, s)|^{q} d s\right\}<\infty
$$

if $1<p<\infty$ and $p^{-1}+q^{-1}=1$, with a similar statement holding for $q=\infty$.

Also, the continuity of the mapping $t \rightarrow a(t, \cdot)$ in the norm topology is equivalent to saying that

$$
\int_{J}|\alpha(t+h, s)-\alpha(t, s)|^{q} d s \rightarrow 0 \text { as } h \rightarrow 0
$$

where $q$ is given as above and a similar statement holds for the case $q=\infty$. Continuity 'n the weak*-topology means that for each $x$ in $\mathfrak{H B}_{p}(J)$

$$
\left.\mid \int_{J} a(t+h, s)-a(t, s)\right] x(s) d s \mid \rightarrow 0 \text { as } h \rightarrow 0 .
$$

With $p$ satisfying $1 \leqq p<\infty$ we define $\mathbb{C}=\mathbb{C}(I, W)$ as the collection of of all functions $f$ that satisfy Hypothesis $A ; \mathfrak{G}_{p}$ as the collection of all func. tions $g$ that satisfy Hypothesis $B_{p}$; and $\mathfrak{A}_{p}$, or $\mathfrak{Z}_{p}^{*}$, as the collection of all functions a that satisfy Hypothesis $C_{p}$, or $C_{p}^{*}$, respectively. 
We introduce topologies on $\mathcal{C}, \mathfrak{S}_{p}, \mathfrak{A}_{p}$ and $\mathfrak{A}_{p}^{*}$ as follows:

On $\mathcal{C}$ we shall use the topology of uniform convergence on compact sets. This topology is metrizable. For a discussion of this ef. G. R. SELL [13].

On $\mathfrak{G}_{p}$ we define two topologies $\mathscr{G}_{c}$ and $\mathscr{G}_{b}$. We say that $g_{n} \rightarrow g$ in $\mathscr{G}_{c}$ if for each compact interval $J \subset I$ and each compact set $l_{k} \subset \mathbb{C}(J, W)$ the gene. ralized sequence $\left\{g_{n}(x(\cdot), \cdot)\right\}$ converges in $\mathfrak{I}_{p}\left(J, R^{n}\right)$ to $g(x(\cdot), \cdot)$ with covergence uniform for $x(\cdot) \in \mathbb{l} k$.

We say that $g_{n} \rightarrow g$ in $\widetilde{G}_{b}$ if for every compact interval $J \subset I$ and every compact set $K \subset \mathrm{W}$ the generalized sequence $\left.\left\{g_{n}(x)(\cdot), \cdot\right)\right\}$ converges to $g(x) \cdot(, \cdot)$ in $\mathfrak{I}_{p}\left(J, R^{n}\right)$ uniformly for $x(\cdot) \in \mathbb{K}$ where

$$
\mathbb{k}=\mathbb{C}(J, K)=\left(x \in \mathbb{C}\left(J, R^{n}\right) ; x(t) \in K \text { for all } t \in J\right)
$$

The difference between the two topologies $\widetilde{G}_{c}$ and $\widetilde{G}_{b}$ can easily be seen in the case $W=R^{n}$. For both topologies we have the defining condition

$$
\sup _{x(.) \in \mathbb{k}} \int_{j}\left|g_{n}(x(t), t)-g(x(t), t)\right|^{p} d t \rightarrow 0,
$$

For $\widetilde{G}_{c}$ the set $\|_{k}$ is required to be compact while for $\widetilde{\sigma}_{b}$ the set $\mathbb{l} k$ is required to be bounded. The topology $\tau_{b}$ is metrizable, and $\tau_{c}$ is a uniform topology.

On $\mathfrak{A}_{p}$, or $\mathfrak{A}_{p}^{*}$, we say that a generalized sequence $\left\{a_{n}\right\}$ converges to a limit $a$ if for every compact interval $J \subset I$ the sequence $\left\{a_{n}(t, \cdot)\right\}$ converges to $a(t,$.$) in the norm or, respectively, the weak*-topology on \mathfrak{J B}_{p}^{*}(J)^{n}$, uniformly for $t \in J$.

We are interested in the existence, uniqueness and continuity of solutions of $x(t)$ of the integral equation

$$
x(t)=f(t)+\int_{0}^{t} a(t, s) g(x(s), s) d s .
$$

We say that $x(t)$ is solution of (3.p) if it is measurable, satifies (3.p) on some interval $[0, \alpha)$ and is bounded on compact sub-intervals of $[0, \alpha)$. Before giving the main results, let us make note of two lemmas. Each of these lemmas are easily verified.

Lemia 1. - Let $f \in \mathbb{C}, g \in \mathfrak{S}_{p}$ and $a \in \mathfrak{H}_{p}^{*}, 1 \leqq p<\infty$. If there exists a solution $x$ of $(3 . \mathrm{p})$ on the interval $[0, a)$, then $x$ is a continuous function.

LemMa 2. - Let $x(t), 0 \leqq t \leqq \alpha$, be a solution of (3.p) and let $\xi(t), 0 \leqq t \leqq \beta$ be a solution of

$$
\xi(t)=\bar{f}(t)+\int_{0}^{t} a(t+\alpha, s+\alpha) g(\xi(s), s+\alpha) d s,
$$


where

$$
\bar{f}(t)=f(t+\alpha)+\int_{0}^{\alpha} a(t+\alpha, s) g(x(s), s) d s .
$$

Then

$$
x(t)=\left\{\begin{array}{l}
x(t), 0 \leqq t \leqq \alpha \\
\xi(t-\alpha), \alpha \leqq t \leqq \alpha+\beta
\end{array}\right.
$$

is a solution of (3.p) on $0 \leqq t \leqq \alpha+\beta$.

\section{3. - Existence and uniqueness.}

In this section we state and prove our main results on the existence and uniqueness of solutions. The first theorem is an existence theorem. It also contains the generalization of H. KNESER's [5] theorem to integral equations. The question of continuous dependence on $f, g$ and a is treated in the next section.

Theorem 1. - Let $f \in \mathbb{C}, g \in \mathfrak{S}_{p}$ and $a \in \mathfrak{Z}_{p}, 1 \leqq p<\infty$.

(A) Then there exists an interval $[0, \alpha), \alpha>0$, and $a$ continuous func. tion $x:[0, \alpha) \rightarrow W$ such that (3.p) is satisfied for $0 \leqq t<\alpha$.

(B) If $[0, \alpha$ ) denotes the maximal interval of definition of $x$ (which means that the solution $x$ cannot be continued to the right of $\alpha$ ), then either $\alpha$ is a boundary point of $I$ or $x(t) \rightarrow b d y W$ as $t \rightarrow \alpha$.

(C) There is an $\widehat{\alpha}>0$ such that for each $t, 0 \leqq t<\widehat{\alpha}$, the cross-section

$$
K_{t}=\{y \in W: y=x(t) \text { where } x \text { is some solution of (3.p) }\}
$$

is compact. Moreover $\bar{\alpha}$ can be chosen to be maximal in the sense that $\bar{\alpha}=\alpha$, where $\alpha$ is given by $(\mathrm{B})$ for some solution $x$.

Proof. - We shall give an argument for the case where $1<p<\infty$. The proof for the ease $p=1$ differ from this only in the form of some of the equations.

The first part of the theorem is an existence theorem. We shall prove this by applying the Schander-Tychonoff Fixed Point Theorem to the operator $T$ defined formally by $y=T x$ where

$$
y(t)=f(t)+\int_{\varphi}^{t} a(t, s) g(x(s), s) d s .
$$


R. K. Miller - G. R. Sell: Existence, uniqueness and continuity, etc.

141

We want to show that $T$ has a fixed point.

Choose $\beta>0$ so that $[0, \beta] \subset I$. By the usual arguments one can find an $\varepsilon>0$ so that the set

$$
\mathbb{D}[0, \beta]=\{x(\cdot) \in \mathbb{C}([0, \beta], W):|f(t)-x(t)| \leq \varepsilon \text { for } 0 \leq t \leq \beta\}
$$

is a closed convex set in the Banach space $\mathbb{C}\left([0, \beta], R^{n}\right)$. We shall now show that there is a $\beta^{\prime}, 0<\beta^{\prime} \leqq \beta$, such that $T$ maps $\mathbb{D}\left[0, \beta^{\prime}\right]$ into itself.

First we define

$$
B=\sup _{0 \leq t \leq \beta}\left(\int_{0}^{\beta}|a(t, s)|^{q} d s\right)^{\frac{1}{q}}
$$

where $p^{-1}+q^{-1}=1$. By Hypothesis $B_{p}$ there is a function $m$ such that $|g(x, t)| \leq m(t)$, for $x \in K$ and $0 \leq t \leq \beta$, where

$$
\int_{0}^{\beta} m^{p} d t<\infty
$$

Now choose $\beta^{\prime}, 0<\beta^{\prime}, \leqq \beta$ so that

$$
B\left(\int_{0}^{\beta^{\prime}} m^{p} d t\right)^{\frac{1}{p}} \leqq \varepsilon .
$$

We then claim that $T$ maps $\mathbb{\Phi}\left[0, \beta^{\prime}\right]$ into itself. Indeed, if $x \in \Phi\left[0, \beta^{\prime}\right]$ and $0 \leqq t \leqq \beta^{\prime}$ then by $(2 . \mathrm{p})$ we get

$$
\begin{aligned}
|y(t)-f(t)| & =\left|\int_{0}^{t} a(t, s) g(x(s), s) d s\right| \\
& \leqq\left\{\int_{0}^{t}|a(t, s)|^{q} d s\right\}^{\frac{1}{q}}\left\{\int_{0}^{t}|g(x(s), s)|^{p} d s\right\}^{\frac{1}{p}} \\
& \leqq B\left\{\int_{0}^{t} m(s)^{p} d s\right\}^{\frac{1}{p}} \leqq \varepsilon
\end{aligned}
$$

hence $y \in \mathbb{\boxplus}\left[0, \beta^{\prime}\right]$.

Now we shall show that $T$ is compact. For this purpose it suffices to show that the set of functions $T\left(\mathbb{P}\left[0, \beta^{\prime}\right]\right)$ is equi-continuous. Let $t$ be fixed 
with $0 \leq t \leq \beta^{\prime}$ and let $\varepsilon>0$ be given. Then

$$
\begin{aligned}
& |T x(t+h)-T x(t)| \leqq|f(t+h)-f(t)|+\left|\int_{t}^{t+h} a(t+h, s) g(x(s), s) d s\right| \\
& \left.+\left|\int_{0}^{t}[a \mid t+h, s)-a\right| t, s\right) \mid g(x(s), s|d s| \\
& \leqq|f(t+h)-f(t)|+\left\{\int_{t}^{t+h}|a(t+h, s)| q d s \mid\right\} \mid \frac{1}{q}\left\{\left|\int_{t}^{t+h}\right| g(x(s), s)|p d s|\right\}^{\frac{1}{p}} \\
& +\left\{\int_{0}^{t}|a(t+h, s)-a(t, s)|^{q} d s\right\}\left\{\frac { 1 } { q } \left\{\left.\int_{0}^{t}|g(x(s), s)|^{p} d s\right|^{\frac{1}{p}}\right.\right. \\
& \leqq|f(t+h)-f(t)|+B\left\{\left|\int_{t}^{t+h} m(s)^{p} d s\right|\right\}^{\frac{1}{p}} \\
& +\left\{\int_{0}^{p^{\prime}}\left|a(t+h, s)-a\left({ }^{\prime}, s\right)\right|^{\alpha} d s\right\}^{\frac{1}{q}}\left\{\int_{0}^{p^{\prime}} m(s)^{p} d s\right\}^{l^{\mathrm{L}}} \text {. }
\end{aligned}
$$

Now choose $\delta>0$ so that if $|h| \leqq \delta$ then

$$
\begin{gathered}
|f(t+h)-f(t)| \leqq \varepsilon \\
B \mid \int_{t}^{t+h} m\left(\left.s\right|^{p} d s \mid \frac{1}{p} \leqq \varepsilon\right. \\
\left\{\int_{0}^{\beta^{\prime}} \mid a(t+h, s)-a\left(t,\left.s\right|^{q} d s\right\} \frac{1}{\alpha}\left\{\int_{0}^{\beta^{\prime}} m s\right)^{p} d s\right\}^{\frac{1}{p}} \leqq \varepsilon .
\end{gathered}
$$

Note that $\delta$ depends on $t$ and $\varepsilon$ but it is independent of the function $x$. It follows then that

$$
|T x(t+h)-T x(t)| \leqq 3 \varepsilon \quad(|h| \leqq \delta)
$$

which shows that $T$ is compact.

Now we shall show that $T$ is continuous. If $\left\{x_{n}\right\}$ is a generalized sequence in $\mathbb{\Phi}\left[0, \beta^{\prime}\right]$ with limit $x$, then by the continuity of $g(x, l)$ in $x$ we get

$$
g\left(x_{n}(s), s\right) \rightarrow g(x(s), s)
$$


for each $s, 0 \leqq s \leqq \beta^{\prime}$, and

$$
a(t, s) g\left(x_{i z}(s), s\right) \rightarrow a(t, s) g(x(s), s) .
$$

Furthermore, the generalized sequence $\left\{a(t, s) g\left(x_{n}(s), s\right)\right\}$ is bounded by an integrable function,

$$
\left|a(t, s) g\left(x_{n}(s), s\right)\right| \leqq|a(t, s)| m(s)
$$

for $n$ sufficiently large, say $n \geq N$, hence by Lebesgue's Theorem

$$
\int_{0}^{t} a(t, s) g\left(x_{n}(s), s\right) d s \rightarrow \int_{0}^{t} a(t, s) g(x(s), s) d s .
$$

This implies that for each $t, 0 \leqq t \leqq \beta^{\prime}$, one has

$$
T x_{n}(t) \rightarrow T x(t) \text { as } n \rightarrow \infty \text {. }
$$

In order to show that $T$ is continuous we must show that the convergence in (5) is uniform. However, this follows easily from the fact that $T x$ is continuous and the set $\left\{T x_{n}\right\}$ is equi-continuous.

We have thus shown that $T$ is a compact, continuous operator, therefore by the Schauder-Tychonoff Fixed Point Theorem, ef. Cronin [3; p. 131], T has a fixed point $x$.

Let us now show that the maximal interval of definition $[0, \alpha)$ is charac. terized in the form described in the theorem. Proceeding by contradiction, assume that $\alpha$ is not a boundary point of $J$ and that $x(t)$ remains in a compact set $K \subset W$ for $0 \leqq t<\alpha$. We will then show that there is a solution $\bar{x}(t)$ of (1) defined on an interval $\left[0, \alpha^{\prime}\right)$ where $\alpha<\alpha^{\prime}$ and such that $\bar{x}(t)=x(t)$ for $0 \leqq t<\alpha$. This will contradict the maximality of $[0, \alpha)$.

The first step is to show that $\lim x(t)$ exists. We shall call this limit $x(\alpha)$. The limit exists if for every $\varepsilon>0$ there is a $\tau, 0<\tau<\alpha$ such that $|x(t)-x(u)| \leqq \varepsilon$ for all $t$ and $u$ with $\tau \leqq t<\alpha, t \leqq u<\alpha$

By Hypothesis $B_{p}$, there is a function $m$ such that $|g(x, t)| \leqq m(t)$ for $x \varepsilon K$ and $0 \leqq t \leqq \alpha$, where

$$
\begin{gathered}
\int_{0}^{x} m^{p} d s<\infty . \\
\text { If } \tau \leqq t \leqq u<\alpha, \text { then } \\
|x(u)-x(t)| \leqq|f(u)-f(t)|+\mid \int_{0}^{t}\{a(t, s)-a(u, s)|g(x(s), s) d s|
\end{gathered}
$$




$$
\begin{aligned}
& +\left|\int_{i}^{u} a(u, s) g(x(s), s) d s\right| \\
& \leqq|f(u)-f(t)| \\
& +\left\{\int_{0}^{\alpha}|a(t, s)-a(u, s)|^{q} d s\right\}^{\frac{1}{q}}\left\{\int_{0}^{\alpha} m(s)^{p} d s\right\}^{\frac{1}{p}} \\
& +\left\{\int_{0}^{\alpha}|a(u, s)|^{p} d s\right\}^{\frac{1}{\alpha}}\left\{\int_{\tau}^{\alpha} m(s)^{p} d s\right\}^{\frac{1}{p}} .
\end{aligned}
$$

Thus our assumptions clearly im $\operatorname{im}_{1}$ ly that if $\tau \leqq t \leqq u<\alpha$ and $\alpha-\tau$ is sufficiently small, then $|x(u)-x(t)|<\varepsilon$. Thus we see that $x(t)$ is a solution of (3.p) on the closed interval $[0, \alpha]$.

Now by applying the previous existence proof with Lemma 2 , we conclude that the solution $x$ can be continued for $0 \leqq t \leqq \alpha+\beta, \beta>0$, and this contradicts the maximality of $[0, \alpha)$.

The proof that the cross-sections are compact is simple modification of Kneser's Theorem for ordinary differential equations, cf. G. R. SELL [12, p, 373]. The critical thing to show is that if $\left\{x_{n}\right\}$ is a generalized sequence of solu. tions of (3.p) that converges uniformly on compact sets to a function $x$, then $x$ is a solution of (3.p). This, however, is a direct application of the Lebesgue Dominated Convergence Theorem, which completes the proof. The fact that $\alpha$ is maximal in the sense indicated can also be proved with the same techniques, of. $[12 ;$ p. 382].

REMARKs 1. - As noted in the Introduction, the Scauder-Tychonoff Fixed Point Theorèm has been used before to get existence criteria for integral equations. See, for example, $[2,3,6]$. The fact that the maximal interval of definition is characterized by Statement (B) has been proved by essentially the same argument but under more restrictive conditions by J. A. NoHEL [8]. Finally a special case of (C), which generalizes Kneser's Theorem, has been proved by T. SATö [11].

2. - Our argument does break down if we replace the Hypothesis $C_{p}$ for $a(t, s)$ be the weaker Hypothesis $C_{p}^{*}$. The only place where the stronger hypothesis was used to show that the operator $T$ is compact. We have no counter example to show that Theorem 1 is false under the the weaker hypothesis on $a(t, s)$.

In order to get uniqueness of solutions, we impose a Lipschitz condition on g. Actually, if $g$ satisfies a Lipschitz condition, then we can relax the assumption, on the kernel a and ask that it satisfy Hypothesis $C_{p}^{*}$. 
THEOREM 2. - Let $f \in \mathbb{C}, g \varepsilon \mathcal{S}_{p}$ and $a \in \mathfrak{A}_{p}^{*}, 1 \leqq p<\infty$, and assume that $g$ satisfies a Lipschitz condition. Then there existes one and only one solution of (3.p). Furthermore the maximal interval of definition is characterized by (B) of Theorem 1.

The proof of this is completely straightforward. One proves, by the usual arguments, cf. [8], that the operator $T$ is a contraction on some set $\mathbb{D}\left[0, \beta^{\prime \prime}\right]$. $W_{\theta}$ omit the details.

REMARK. - One can replace the Lipschitz condition on $g$ with a weaker statement. For example, one could replace it with the Osgood condition:

where $\int_{0}^{\alpha} \frac{d r}{\psi(r)}=+\infty$. Comparison theorems of this type are well-known for

$$
|g(x, t)-g(y, t)| \leqq k(t) \psi(|x-y|)
$$

differential equations and they have been used for integral equations, ef. $[8,11]$.

\section{4. - Continuity of solutions.}

In this section we investigate the dependence of the solutions $x$ on the three therms $f, g$ and $\alpha$.

Theorem 3. - Let $\left\{f_{n}\right\},\left\{g_{n}\right\}$ and $\left\{a_{n}\right\}$ be generalized sequence in $\mathbb{C}, \mathfrak{G}_{p}$ and $\mathfrak{A}_{p}$ respectively where $1 \leqq p<\infty$. Assume that these sequences have limits $f_{n} \rightarrow f, g_{n} \rightarrow g\left(i n \widetilde{\sigma}_{c}\right)$ and $a_{n} \rightarrow a$ in the respective spaces. Let $\left\{x_{n}\right\}$ be a sequence of solutions of

$$
x_{n}(t)=f_{n}(t)+\int_{0}^{t} a_{n}(t, s) g_{n}\left(x_{n}(s), s\right) d s
$$

on the maximal intervals $\left[0, \alpha_{n}\right)$, Then the sequence $\left\{x_{n}\right\}$ has a uniformly convergent subsequence on some interval $0 \leqq t \leqq \sigma, \sigma>0$. The limit function $x$ is a solution of the limiting equation

$$
x(t)=f(t)+\int_{0}^{t} \alpha(t, s) g(x(s), s) d s .
$$

Moreover, the subsequence $\left\{x_{n_{j}}\right\}$ of $\left\{x_{n}\right\}$ may be chosen so that $x_{n_{j}}(t) \rightarrow x_{i}(t)$ uniformly on compact subsets of $[0, \bar{\alpha})$, where the interval $[0, \bar{\alpha}]$ is the maximal interval on which the cross section $K_{t}$ of (6.p) are compact (see Theorem $1(\mathrm{C}))$ and $[0, \widehat{\alpha}) \subset \lim$ inf $\left[0, \alpha_{n}\right)$.

Proor. - We will show that for any $\beta$ with $0<\beta<\widehat{\alpha}$, one has:

1. $\left[0, \beta_{]} \subseteq\left[0, \alpha_{n}\right)\right.$ for $n$ sufficiently large, say $n \geq N_{o}$; 
2. on the interval $0, \beta]$ the sequence of functions $\left\{x_{n}\right\}$ is bounded and equicontinuous; and

3. if $\left\{x_{n j}\right\}$ is a convergent subsequence of $\left\{x_{n}\right\}$ with limit $x(t)$ on $[0, \beta]$, then $x(t)$ is a solution of $(6 . p)$ on $[0, \beta]$.

Again we shall prove this for the case $1<p<\infty$. The proof for the case $p=1$ is similar.

Let $\beta, 0 \leqq \beta<\bar{\alpha}$, be given where $\bar{\alpha}$ is given by the hypothesis. Then the cross section

$$
K_{t}=\{y \in W ; y=x(t) \text { for some solution } x \text { of }(6 . p)\}
$$

is a compact subset of $W$ for $0 \leqq t \leqq \beta$. It is easily shown that

$$
\widehat{K}=\cup\left\{K_{t} ; 0 \leqq t \leqq \beta\right\}
$$

is a compact subset of $W$, of. e.g. [12, p. 378]. Let $K$ be a compact set in $W$ that contains $\widehat{K}$ in its interior. By hypothesis $B_{p}$ there is a function $m \in \mathfrak{L}_{p}[0, \beta]$ such that

$$
|g(x, t)| \leqq m(t) . \quad(x \in K, 0 \leqq t \leqq \beta)
$$

The convergence $g_{n} \rightarrow g$ in $\widetilde{\sigma}_{c}$ implies that $\varepsilon_{n} \rightarrow 0$ where

$$
\varepsilon_{n}=\sup _{x \in K} \int_{0}^{\beta}\left|g_{n}(x, s)-g(x, s)\right|^{p} d s .
$$

Furthermore if $0 \leqq \sigma \leqq \beta$, then

$$
\begin{aligned}
\left\{\int_{0}^{\sigma}\left|g_{n}(x, s)\right| p d s\right\}^{\frac{1}{p}} \leqq & \left\{\int_{0}^{\sigma}\left|g_{n}(x, s)-g(x, s)\right| p d s\right\}^{\frac{1}{p}}+\left\{\int_{0}^{\sigma} m\left(\left.s\right|^{p} d s\right\}^{\frac{1}{p}}\right. \\
& \leqq \varepsilon_{n}+\left\{\int_{0}^{\sigma} m(s)^{p} d s\right\}^{\frac{1}{p}} .
\end{aligned}
$$

For $\sigma$ in the interval $[0, \beta]$ set

$$
M(\sigma, n)=\varepsilon_{n}+\left\{\int_{0}^{\sigma} m(s)^{p} d_{k} s\right\}^{\frac{1}{p}}
$$

Similarly we can find a common bound for the sequence $\left\{a_{n}\right\}$, that is

$$
\left.B=\sup _{n}\left[\sup _{0 \leqq t \leqq P}\left\{\int_{0}^{R}\left|a_{n}(t, s)\right| q d s\right\}^{1}\right\}^{1}\right]<\infty
$$


where $p^{-1}+q^{-1}=1$. Note that the bound $B$ in (8) also holds for the limiting function $a(t, s)$.

By the choice of the set $K$, there is an $\varepsilon>0$ such that if $x(t)$ is any solution of (6.p), $0 \leqq t \leqq \beta$, and $|y-x(t)| \leqq \varepsilon$ then $y \in K$. Fix $\delta$ so that $0<\delta<\varepsilon / 2$ and fix the index $N_{1}$ so that if $n \geqq N_{1}$ then $2 B \varepsilon_{n}<\delta$ and

$$
\left|f_{n}(t)-f(t)\right|<\delta . \quad\left(0 \leqq t \leqq \beta, n \geqq N_{1}\right)
$$

Now choose $\sigma$ so that $0<\sigma \leqq \beta$ and

$$
2 B\left\{\int_{0}^{\sigma} m(s)^{p} d s\right\}^{\frac{1}{p}}=\varepsilon-2 \delta .
$$

If this equality cannot be satisfied for $0 \leqq \sigma \leqq, \beta$, then choose $\sigma=\beta$.

We will now show that on the interval $[0, \sigma]$ one has $x_{n}(t) \in K$ for all $n \geqq N_{1}$. Let $x(t)$ be any solution of $(6 . p)$ defined on $[0, \sigma]$. we shall show that $\left|x_{n}(t)-x(t)\right| \leqq \varepsilon$ for $0 \leqq t \leqq \sigma$ and $n \geqq N_{1}$. For $t=0$ w $\theta$ have

$$
\left|x_{n}(0)-x(0)\right|=\left|f_{n}(0)-f(0)\right|<\delta<\varepsilon .
$$

Suppose $\left|x_{n}(t)-x(t)\right| \leqq \varepsilon$ for $0 \leqq t<\xi \leqq \sigma$. Then

$$
\begin{gathered}
\left|x_{n}(\xi)-x(\xi)\right| \leqq \\
\left|f_{n}(\xi)-f(\xi)\right|+\left|\int_{0}^{\xi} a_{n}(\xi, s) g_{n}\left(x_{n}(s), s\right) d s\right| \\
+\left|\int_{0}^{\xi} a(\xi, s) g(x(s), s) d s\right| .
\end{gathered}
$$

Applying (2.p), (7) and (8) we see tha

$$
\begin{aligned}
\left|x_{n}(\xi)-x(\xi)\right| & \leqq \delta+2 B M(\xi, n) \\
& \leqq \delta+2 B M(\sigma, n)<\varepsilon .
\end{aligned}
$$

Hence the maximal interval $[0, \xi]$ for which $x_{n}(t) \in K$ if $0 \leqq t<\xi$ must include $[0, \sigma]$.

We shall now show that on the interval $[0, \sigma]$, the sequence of functions $\left\{x_{n} n \geqq N_{1}\right\}$ is equicontinuous. If $0 \leqq t \leqq t+h \leqq \sigma$, then

$$
\begin{gathered}
\left|x_{n}(t+h)-x_{n}(t)\right| \leqq\left|f_{n}(t+h)-f_{n}(t)\right| \\
+\left|\int_{0}^{t}\left\{a_{n}(t+h, s)-a_{n}(t, s)\right\} g_{n}\left(x_{n}(s), s\right) d s\right|
\end{gathered}
$$




$$
\begin{aligned}
& +\mid \int_{t}^{t+h} a_{n}\left(t+h, s\left|g_{n}\left(x_{n}(s), s\right) d s\right|\right. \\
& \leqq\left|f_{n}(t+h)-f_{n}(t)\right|+B\left\{\int_{t}^{t+h} m(s)^{p} d s \frac{1}{p}\right. \\
& +\left\{\int_{0}^{\sigma}\left|a_{n}(t+h, s)-a_{n}(t, s)\right| q d s\right\}^{\frac{1}{\alpha}} M(\sigma, n) .
\end{aligned}
$$

Siuce $f_{n} \rightarrow f$ uniformly on $[0, \sigma]$, the sequence $\left\{f_{n}\right\}$ is equicontinuous. Since $a_{n}(t, \cdot) \rightarrow a(t, \cdot)$ uniformly in $t \in[0, \sigma]$, it follows that the sequence $\left\{a_{n}\right\}$ is equicontinuous as functions of $t$ with values in $\mathfrak{B}_{q}[0 \text {, } \sigma]^{n}$. Since $B$ is a fixed constant and $M(\sigma, n)$ is bounded in $n$, for $n \geq N_{1}$, we see that $\left\{x_{n} ; n \geqq N_{1}\right\}$ is equicontinuous on $[0, \sigma]$.

Now choose any convergent subsequence of $\left\{x_{n}\right\}$. To simplify the notation we shall write $\left\{x_{n}\right\}$ for this subsequence. Then there is a function $x$ such that $x_{n}(t) \rightarrow x(t)$ uniformly on $[0, \sigma]$. Since

$$
\mathbb{T}=\left\{x, x_{1}, x_{2}, x_{3}, \ldots\right\},
$$

is a compact set in $\mathbb{C}([0, \sigma], W)$ and $g_{n} \rightarrow g$ in $\mathcal{G}_{c}$ it follows that

$$
\left.\left.g_{n}\left(x_{n}(\cdot), \cdot\right) \rightarrow g(x) \cdot \cdot\right) \cdot\right)
$$

in $\mathfrak{L}_{p}\left[[0, \sigma], R^{n}\right)$. Also we have

$$
a_{n}\left(t, \cdot g_{n}\left(x_{n}(\cdot), \cdot\right) \rightarrow a(t, \cdot) g(x \mid \cdot), \cdot\right)
$$

in $\mathfrak{L}_{1}\left([0, \sigma], R^{n}\right)$ for $0 \leqq t \leqq \sigma$. Thus

$$
\int_{0}^{t} a_{n}(t, s) g_{n}\left(x_{n}(s), s\right) d s \rightarrow \int_{0}^{t} \alpha(t, s) g(x(s), s) d s .
$$

It follows that $x(t)$ satisfies $(6 . p)$ on $[0, \sigma]$.

We now want to show that the interval $[0, \sigma]$ can be extended to $[0, \beta]$. This extension can be performed in a finite number of repetition of the above argument. That is, consider the translation of (6.p) given by

$$
X(t)=\vec{f}(t)+\int_{0}^{t} a(t+\sigma, s+\sigma) g(X(s), s+\sigma) d s
$$


where $\widehat{f}(t)=f(t+\sigma)+\int_{0}^{\sigma} a(t+\sigma, s \mid g(x(s), s) d s$. Equation (5.p) is similarly tran. slated. By the above argument one can find a $\tau>0$ such that a subsequence of the solutions $\left\{X_{n}(t)\right\}$ converge to a solution $X(t)$ of $(9 . p)$ on $[0, \tau]$. By Lemma 2, we see that

$$
x(t)= \begin{cases}x(t) & 0 \leqq t \leqq \sigma \\ X(t-\sigma) & 0 \leqq t \leqq \sigma+\tau\end{cases}
$$

is a solution of (6.p) on $[0, \sigma+\tau]$, and it is the limit of a subsequence of $\left\{x_{n}(t)\right\}$ on $0 \leqq t \leqq \sigma+\tau$.

This process can now be repeated. In order to show that one can extend to $[0, \beta]$ in a finite number of steps, it is necessary to keep track of the size of each step. This is governed by the function $M(\sigma, n)$ defined above. That is, the number $\tau$ can be chosen so that $0<\tau \leqq \beta-\sigma$ and

$$
2 B\left\{\int_{\sigma}^{\sigma+\tau} m(s)^{p} d s\right\}^{\frac{1}{p}}=\varepsilon-2 \delta,
$$

or if this last equality cannot be satisfied then set $\tau=\beta-\sigma$. Since the integral $\int_{0}^{\beta} m(s)^{p} d s$ is finite, it is clear that one can extend $[0, \sigma]$ to $[0, \beta]$ in a finite number of steps. This completes the proof of Theorem 3.

In the last theorem we assumed that the kernels $\left\{a_{n}(t, s)\right\}$ and the limiting kernel $a(t, s)$ satisfy Hypothesis $C_{p}$ and that $a_{n} \rightarrow a$ in the norm topology, uniformly for $t$ on compact sets $J \subset I$. One can ask whether the weaker convergence would suffice. The answer is yes if one strengthens the convergence on $\left\{g_{n}\right\}$. More precisely we prove the following results :

Theorem 4. - Let $\left\{f_{n}\right\},\left\{g_{n}\right\}$ and $\left\{a_{n}\right\}$ be generalized sequences in $\mathfrak{C}, \mathfrak{S}_{p}$ and $\mathfrak{A}_{p}^{*}$ respectively where $1 \leqq p<\infty$. Assume that the sequences have limits $f_{n} \rightarrow f ; g_{n} \rightarrow g\left(i n \widetilde{\sigma}_{b}\right)$ and $a_{n} \rightarrow a\left(i n \mathfrak{A}_{p}^{*}\right)$ with $f, g$ and $a$ in the respective spaces. Assume further that $g_{n}$ and $g$ satisfy Lipschitz conditions. Let $x_{n}$ be the solutions of

$$
x_{n}(t)=f_{n}(t)+\int_{0}^{t} a_{n}(t, s) g\left(x_{n}(s), s\right) d s,
$$

on the maximal intervals $\left[0, \alpha_{n}\right)$. Then the sequence $\left\{x_{n}\right\}$ converges uniformly on compact subsets of $[0, \alpha)$ to a function $x(t)$. The function $x(t)$ is the unique solution of the limiting equation

$$
x(t)=f(t)+\int_{0}^{t} a(t, s) g(x(s), s) d s
$$

defined on the maximal interval $[0, \alpha)$. Moreover, $\alpha \leqq \lim$ inf $\alpha_{n}$. 
Proof. - For any $\beta, 0<\beta<\alpha$, we shall show that $\alpha_{n} \geqq \beta$ for $n$ sufficiently large (say $n \geqq N_{0}$ ) and that $x_{n}(t) \rightarrow x(t)$ uniformly on $[0, \beta]$. This will prove the theorem.

Fix any $\beta \in[0, \alpha)$. Let $K$ be a compact subset of $W$ that contains the curve $x(t): 0 \leqq t \leqq \beta$ in its interior. Let $m \in \mathbb{I}_{p}\left([0, \beta], R^{n}\right)$ with

$$
|g(x, t)| \leqq m(t) . \quad(x \in K, 0 \leqq t \leqq \beta)
$$

Since the kernels $a_{n}(t, \cdot)$ converge to $a(t, \cdot)$ in the weak*-topology on $\mathfrak{J B}_{q}[0, \beta]^{n}$, they are bounded in the norm topology. Furthermore since the convergence is uniform for $t$ on compact sets, the number $B$ defined by

$$
B=\sup _{n}\left\{\sup _{0 \leq t \leq \beta}\left\{\int_{0}^{\beta}\left|a_{n}(t, s)\right| q d s\right\}^{\frac{1}{q}}\right\}
$$

is finite. Let $M(\sigma, n), \varepsilon, \delta, N_{1}$ and $\sigma$ be defined as in the first part of the proof of Theorem ?

Instead of showing the equicontinuity of $\left\{x_{n}\right\}$ on $[0, \sigma]$ we proceed directly to estimate $\left|x_{n}(t)-x(t)\right|$. Define $R_{n}(t)$ by

$$
\begin{aligned}
R_{n}(t)=\mid f_{n}(t) & -f(t)\left|+\int_{0}^{t}\right| a_{n}(t, s)|| g_{n}\left(x_{n}(s), s\right)-g\left(x_{n}(s) ; s\right) \mid d s \\
+ & \left|\int_{0}^{t}\left\{a_{n}(t, s)-a(t, s)\right\} g(x(s), s) d s\right|
\end{aligned}
$$

and let $\varepsilon_{n}=\sup \left\{R_{n}(t) ; 0 \leqq t \leqq \sigma\right\}$. Because of the convergence assumptions on $\left\{f_{n}\right\},\left\{a_{n}\right\}$ and $\left\{g_{n}\right\}$ and the fact that $x_{n}(t) \in K$ for $0 \leqq t \leqq \sigma, n \geqq N_{1}$ one has $\varepsilon_{n} \rightarrow 0$.

Since $g$ satisfies a Lipschitz condition, there is a function $k(t) \in \mathfrak{U}_{p}\left([0, \beta], R_{n}\right)$ such that

$$
|g(x, t)-g(y, t)| \leqq k(t) \mid x-y ! \cdot \quad(x, y \in K, 0 \leqq t \leqq \beta)
$$

Choose $\sigma^{\prime}, 0<\sigma^{\prime} \leq \sigma$, so that $K_{0}=\left\{\int_{0}^{\sigma^{\prime}} k(t)^{p} d t\right\}^{1 / p}<1 / B$.

By a straight forward computation we get

$$
\begin{aligned}
\left|x_{n}(t)-x(t)\right| & \leqq R_{n}(t)+\int_{0}^{t}\left|a_{n}(t, s)\right|\left|g\left(x_{n}(s), s\right)-g(x(s), s)\right| d s \\
& \leqq \varepsilon_{n}+\int_{0}^{t} l(s)\left|a_{n}(t, s)\right|\left|x_{n}(s)-x(s)\right| d s .
\end{aligned}
$$


By using Hölder's inequality and restricting $t$ to $0 \leq t \leq \sigma^{\prime}$ it is easily shown that

$$
\left|x_{n}(t)-x(t)\right| \leq\left(1-B K_{0}\right)^{-1} \varepsilon_{n} \rightarrow 0 .
$$

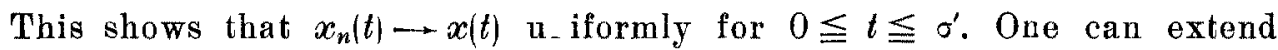
$\left[0, \sigma^{\prime}\right]$ to $[0, \beta]$ by the same reasoning process used in the proof of Theorem 3 , which completes the proof of Theorem 4 .

ReMaris: 1. - The assumption that the limit function $g(x, t)$ satisfies a Lipschitz condition can be weakened. One could use an Osgood condition or a comparison theorem used by J. NoHeL [8] or T. SaTó [11]. However, it does not appear that in Theorem 5 one ean drop this type of analytical criterion, which implies uniqueness, and assume directly that the solutions are unique.

2. - It should be noted that E. KaMkE's Theorem [4] on the continuity of solutions of ordinary differential equations, as well as Z. OpIaL's generalization [9] are included as special cases of Theorem 4. In these papers the kernel $a(t, s)$ reduces to the identity matrix. KAMkw assumed that the functions $g_{n}(x, t)$ and $g(x, t)$ were continuous and that $g_{n} \rightarrow g$ uniformly on compact sets. this convergence implies $g_{n} \rightarrow g$ in $\widetilde{\sigma}_{b}$ for every $\mathfrak{G}_{p}, 1 \leqq p<\infty$. Opial assumel that the funetions $g_{n}$ and $g$ satisfied Hypothesis $B_{1}$ and $g_{n} \rightarrow g$ in $\mathscr{C}_{b}$ for $p=1$.

3. - Many variations of our theorems are possible. One variation is of particular interest since many applications fit into this form. For this we set $p=\infty$ and $q=1$. Here we assume $g$ to be continuous in $(t, x)$ and $g_{n} \rightarrow g$ means uniform convergence on compact sets. Suppose now that $a$ satisfies the following conditions:

(i) for each compact interval $J \subset I$ and each $\ell \in I$ the map $S: \mathbb{C}(J, W) \rightarrow R^{n}$ defined by

$$
S: x \rightarrow \int_{J} a(t, s) x(s) d s
$$

is a bounded linear functional, $\mathfrak{H B}_{2}(J)$, and

(ii) the mapping $t \rightarrow a(t, \cdot)$ is continuous in the norm topology on

(iii) for any compact set $J \subset I$,

$$
\lim _{h \rightarrow 0} \int_{t}^{t+h}|a(t, s)| d s=0
$$

uniformly for $t \in J$. 
Under these conditions on $g$ and $a$, the obvious variations of Theorems 1 through 4 are true. We omit a formal statement.

4. - Continuity results of the type given by Theorem 4 have been obtained by Levin and Nohel [15] in a special, scalar example.

\section{REFERENCES}

[1] L. CasARI, Asymptotic bohnvior and stability problems in ordinary differential equations, Second edition, Academic Press, New York, (1963)

[2] C. Corduneanu, Problèmes globaux dans la thérie des équations integrales da Volterra, Ann. Mat. Pura Appl. (4), 67 (1965), pp. 349.363.

[3] J. Cronin, Bixed points and topological degree in nonlinear analysis, Amer. Math. Soe, l'rovidence, R. I, (1964).

[4] E. KАMKE, Zur Theorie der Systeme gewöhnlicher Differentialgleichungen II, Acta. Math. 58 (1982), pp. $57-<5$.

[5] H. KNEsER, $\ddot{U} b e r$ die Lösungen eines Systems gewöhnlicher Differentialgleichungen das der Lipschitzschen Bedingung nicht genügts, Sitzber. Preuss. Akad. Wiss. Phys. Math. Kl. (1923), pp. 171.17ł.

[6] M. A. Krasnosel'sirr, Topological Methods in the Theory of Nonlinear Integral Equations, Pergamon Press, New York, (1:16t).

[7] L. A. Ladyzenskir, Conditions for the complete continuity of P. S. URYson's integral operator in the space of continuous functions, Dokl. Akad. Nauk SSSR 97 (1654). pp. $1105 \cdot 1108$.

[8] J. A. Noнel, Some problems in nonlinenr Volterra integral equations, Bull. Amer. Mat. Soc. 68 (1962), pp. 323-329.

[9] Z. OpraL, Sur la dépendance des solutions d'un système d'équations différentielles de leurs seconds membres. Applications aux systemes presque autonomes, Ann. Polon. Math. 8 (1960), pp. $75 \cdot 89$.

[10] M. REICHERT, Eindeutigkeits-und Interationsfragen bei Volterra-Hammersteinschen Integralgleichungen, J. Reine Angew. Math., 220 (1965), pp. 74-87.

[11] T. Sato-, Sur l'équation intégrale non linéaire de Volterra, Compositio Math., 11 (1958), pp. 271.290 .

[12] G. R. SELt. On the fundamental theory of ordinary differential equations, J. Diff. Equations, 1 (1963), pp. 370.392.

[13] G. R. SELL, Nonautonomous differential equations and topological dynamics $I$. The basic theory, Trans. Amer. Math. Soc. 127 (1967), pp. 241.62.

[14] D. WILLETT, Nonlinear vector integral equations as contraction mappings, Arch. Rational Mech. Anal., 15 (1964), pp. 79.86.

[15] J.J. LEviN and J. A. NOHEL, On a system of integrodifferential equations occurring in reactor dynamics, IT, Archive Rat. Mech. Anal., 11 (1962), pp. 210.243. 